\title{
Diseño de un Sistema de Video Vigilancia en la Plataforma de Telecomunicaciones del Programa Huila Digital para el Municipio de Palermo
}

\section{Design of a Video Surveillance System in the Platform of Telecommunications for Huila Digital Program for the Municipality of Palermo}

\author{
Jesús David Quintero Polanco ${ }^{1}$ y Jesús Antonio Ramos ${ }^{2}$
}

\begin{abstract}
Resumen
En este proyecto, desarrollado para el Municipio de Palermo (Huila - Colombia), se logra la implementación del Sistema de Video Vigilancia con el fin de mejorar la seguridad del Municipio. Este sistema consta de equipos de última tecnología marca Pelco, permitiendo con esto una excelente calidad de imagen de las cámaras, un alcance de visón de hasta 80 metros y ángulos de giro de $360^{\circ}$, lo cual ayuda a realizar un trabajo más eficiente a la Policía en su trabajo de brindar seguridad al Municipio.

Con la implementación de este sistema se está ayudando a mejorar notablemente la seguridad del Municipio y sobre todo se está recuperando la tranquilidad de sus pobladores la cual se había perdido. Otro logro muy importante que se alcanza con la implementación de este sistema, es la instalación para el Municipio de un red de comunicación por fibra óptica, lo cual permite estar a la vanguardia de las comunicaciones y a la vez poder implementar a futuro servicios nuevos que se pueden prestar a través de esta red como lo son: televisión digital, telefonía Ip e Internet.
\end{abstract}

Palabras Clave: Sistema de Video Vigilancia; Tecnologías de comunicación; Televisión Digital; Telefonía Ip; Internet; Fibra óptica; Linux.

\begin{abstract}
In this project, developed for the Municipality of Palermo (Huila - Colombia), it is possible to implement video surveillance system in order to improve the security of the municipality. This system consists of brand-art equipment Pelco, allowing with this excellent quality camera, a range of vision of up to 80 meters and at angles of $360^{\circ}$ turns, which aid has made a more efficient Police in its efforts to provide security to the Municipality. With the implementation of this system is helping to significantly improve the security of the municipality and is recovering all the tranquility of its people which was lost.

Another major milestone reached with the implementation of this system is the facility for the Municipality of a network of optical fiber communication, which can be in the forefront of communications while looking to deploy new services that can be provided over this network are: digital TV, IP telephony and Internet.
\end{abstract}

Keywords: Video Surveillance System, Communication technologies, Digital TV, IP Telephony, Internet, fiber optic Linux.

1 Ingeniero Electrónico. Esp. Teleinformática. Docente Universidad Surcolombiana - Neiva. Av. Pastrana-Carrera 1.jdavid@ usco.edu.co

2 Ingeniero Electrónico, Universidad Surcolombiana - Neiva. Av. Pastrana-Carrera 1.jramoscerquera@gmail.com 


\section{Introducción}

Los sistemas de video vigilancia son aquellos que permiten la gestión de múltiples cámaras de seguridad para el control y supervisión de instalaciones locales y remotas. Se trata básicamente de un grabador digital con capacidad de almacenamiento de imágenes ininterrumpido durante las 24 horas, que muestra los resultados en un monitor. Estos sistemas de video vigilancia se encuentran en versiones para 4, 8 y 16 cámaras. Para la aplicación de estos sistemas deben estar soportados por una tecnología Wireless (inalámbrica) o una Wired (alámbrica).

El video vigilancia generalmente persigue garantizar la seguridad de los bienes y las personas, o se utiliza en entornos empresariales con la finalidad de verificar el cumplimiento por el trabajador de sus obligaciones y deberes laborales. (Agpd, 2006)

Un buen sistema debe proporcionar imágenes de calidad tanto diurnas como nocturnas, ser flexible y fácil de usar y proporcionar imágenes para grabar evidencias o para ayudar al análisis de cualquier incidente. (Rojas, 2007)

La implementación de las tecnologías de la comunicación permitir llegar a la digitalización de las telecomunicaciones, debido a flexibilidad de soportar varios servicios como lo son la Televisión Digital, Telefonía Ip, la Internet y Sistemas de Video Vigilancia, siendo este último el de interés.

Con la versatilidad de estos sistemas y sus excelentes características técnicas de velocidades de transmisión, ancho de banda, seguridad y costo, las hacen muy llamativas para su implementación.

Con los buenos resultados se han realizado este tipo de proyectos en otras ciudades, como se describe a continuación:

El municipio de Campana, en la provincia de Buenos Aires, Argentina, en el año 2009 se implementó un sistema de digitalización con tecnología de Banda Ancha Inalámbrica Motorola. Esta implementación mejoro la eficiencia de la seguridad pública, ofrece diversos servicios a la ciudanía y brinda nuevas aplicaciones en el ámbito de la educación; la Municipalidad de Berazategui, Argentina, en el año 2009 necesitaba interconectar diferentes dependencias municipales y de servicios públicos para mejorar de esta manera las actuales condiciones de funcionamiento de su gestión municipal y atención a sus vecinos, el municipio anunció que la primera etapa de su plan de digitalización había concluido de forma exitosa. Esa primera fase abarcó la interconexión con banda ancha inalámbrica de más de 20 delegaciones y centros de salud, de interés cultural, deportivo y comunitario, un año después, el municipio inaugura su nuevo Centro de Operaciones Municipales, como parte de la segunda etapa de digitalización. La función principal del Centro consiste en recibir las imágenes de 203 cámaras instaladas en lugares claves del Municipio.

La tecnología que permitirá llevar a cabo el monitoreo y la vigilancia es parte de la red de banda ancha inalámbrica.

El fin de este diseño se centra en cumplir con los siguientes objetivos:

- Analizar y evaluar las tecnologías de la comunicación, para encontrar la que mejor se adapta para el municipio de Palermo. (Barbero, 1999)

- Estudio de los requerimientos técnicos para la implementación de un Sistema de Video Vigilancia para uso de una entidad como la Policía.

- Realización del estudio de seguridad del municipio de Palermo para la identificación de los puntos críticos.

El objetivo primario de un Sistema de Video Vigilancia aplicado, es lograr mediante la captación de video y sonido digital el procesamiento de esta información, mediante la adquisición software las siguientes funciones de uso privativo de los organismos de seguridad:

- Reconocimiento de placas

- Reconocimiento facial

- Atención a paquetes extraños

La parte fundamental de este diseño está basado en el estudio de las tecnologías de comunicación, la cual permitirá la selección de una para poder implementar un Sistema de Video Vigilancia, y el estudio y toma de datos de los puntos neurálgicos en cuestiones de seguridad para el Municipio de Palermo. 


\section{Metodología}

2.1 Estudio de seguridad: El diseño del sistema de Video Vigilancia se inicia por medio del estudio de seguridad para el municipio, permitiéndonos con esto la identificación de los sitios a monitorear. Como puntos básicos en los que se debe basar el estudio es con la identificación de: Sitios gubernamentales, lugares de reunión de la población y vías de acceso, y juntamente a estos sitios, se solicita información a la Policía acerca de los barrios más inseguros. Para lograr el objetivo se debe realizar el reconocimiento del municipio para analizar estos sitios y tomar los datos técnicos para cada uno de estos, siendo estos datos técnicos:

1). Distancia del punto elegido hacia donde quedara ubicado el centro de control (para nuestro caso la estación de Policía)

2). Conocer la visibilidad del punto escogido, es decir, si se cuenta con una visión directa hacia el centro de control.

3). En qué punto estratégico se ubicaría cada cámara para lograr una mayor cobertura de captación de imagen.

2.2 Estudio de las tecnologías de comunicación: Debido a que la base para un buen desempeño de un Sistema de Video Vigilancia es el sistema de comunicación, entonces se hace una revisión general de las tecnologías Wireless (inalámbrica) y Wired (alámbrica), para encontrar la que mejor se ajusta según las necesidades.

Descripción de un sistema actual inalámbrico:

- El alcance en rendimiento del sistema actual inalámbrico es de $254 \mathrm{Mbps.}$

- La información puede ser tomada del aire descifrada con cierta facilidad, ya que el estándar IEEE 8002.11i, utilizado para la mayoría de estos sistemas inalámbricos, no es muy robusto y por lo tanto aún tiene mucha vulnerabilidad en ese sector.

- Facilidad de instalación.

- Su funcionamiento depende si existe línea de vista directa.

- Su funcionamiento se afecta por las condiciones climáticas.

- Soporta la implementación de servicios de voz ip e internet.

- Durabilidad de aproximadamente 5 años.

- Costos de instalación moderados

Descripción de un sistema actual alámbrico:

- El alcance en rendimiento del sistema actual alámbrico es de 10 Gbps.

- Gran seguridad en el transporte de datos por tratarse de sistemas cableados.

- Ancho de banda continúo.

- Mayor trabajo en la instalación.

- Soporta servicios de voz ip, televisión digital e internet.

- Mantenimientos preventivos limitados.

- Durabilidad de aproximadamente 10 años.

- Costo de implementación elevada.

Teniendo en cuenta estas especificaciones técnicas de cada una de estas tecnologías y junto con los requisitos de un Sistema de Video Vigilancia para un correcto funcionamiento, se decide por la tecnología alámbrica específicamente la Fibra Óptica debido a los siguientes argumentos:

1). Por cada sistema de 16 cámaras se necesita una velocidad de transmisión de $128 \mathrm{Mbps}$.

2). La línea de vista de algunos puntos es imposible.

3). La seguridad del tráfico de datos es total.

4). Mantenimientos preventivos limitados.

5). Por la durabilidad.

6). Relación costo/beneficio mayor.

Existen dos tipos de fibra óptica, la monomodal y la multimodal, pero se elige la monomodal debido a que ofrece un mayor alcance en distancia de aproximadamente $20 \mathrm{~km}$ a comparación de los $4 \mathrm{~km}$ de la multimodo, y por ofrecer un mayor ancho de banda de aproximadamente $10 \mathrm{Gbps}$ a comparación de los $1.5 \mathrm{Gbps}$ de la multimodal. 


\subsection{Elección de los equipos a utilizar en el sistema de video vigilancia}

\section{a. Cámaras}

Para la elección de este dispositivo se tuvieron en cuenta las siguientes características técnicas:

1). Tipo domo con capacidad de movimiento de $360^{\circ}$.

2). Control PTZ (movimiento vertical, horizontal y acercamiento)

2). Que posea visión día/noche.

3). Alcance de visión de aproximadamente 80mts.

4). Conexión a internet.

Se eligió una Cámara Pelco Spectra IV SE, la cual se adapta a las necesidades requeridas.

\section{b. transmisor/Receptor}

Para la elección de este dispositivo se tuvieron en cuenta las siguientes características técnicas:

1). Transmisión de video digital.

2). Buena potencia de transmisión.

3). Buen alcance de distancia.

Para este sistema se eligió el siguiente dispositivo, el cual se adapta mas a las necesidades buscadas:

Transmisor Pelco FT85011A/Receptor FR85011A

\section{c. Grabador de video digital (DVR)}

Para la elección del grabador de video, se tuvieron en cuenta las siguientes características técnicas:

1). Facilidad de manejo.

2). Capacidad de grabado superior a un mes.

3). Conectividad a internet.

4). Buena resolución de grabación.

Se eligió el Grabador de Video Digital LG LE2116D.

\section{d. Teclado controlador}

Para la elección de este dispositivo se tuvieron en cuenta las siguientes características técnicas:

1). Capacidad de manejo PTZ.

2). Facilidad de manejo.

Para este sistema se eligió el Teclado controlador Pelco KBD200A

\section{e. Monitor}

Para la elección del monitor se tuvieron en cuenta las siguientes características técnicas:

1). Gran dimensión para poder observar mejor los detalles.

2). Alta definición de video.

3). Disponibilidad variada de terminales de salida.

Para este sistema se eligió el siguiente dispositivo, el cual se adapta a las necesidades buscadas: Monitor Samsung LCD 46".

Por último se eligió una UPS tipo Qbex Sedna de 2KVA, que cuenta con respaldo eléctrico de más de 2 horas 


\section{Resultados}

\subsection{Total de sitios a monitorear}

Como resultado del estudio de seguridad se encontró un total de 15 puntos críticos dentro del municipio, los cuales eran los más vulnerables en lo concerniente a la seguridad.

\subsection{Diseño final de sistema de video vigilancia}

Como diseño final de este sistema Teniendo en cuenta las características del sistema que se desea, la ubicación de los puntos a monitorear y la tecnología de comunicación elegida, se obtiene el siguiente diseño final:

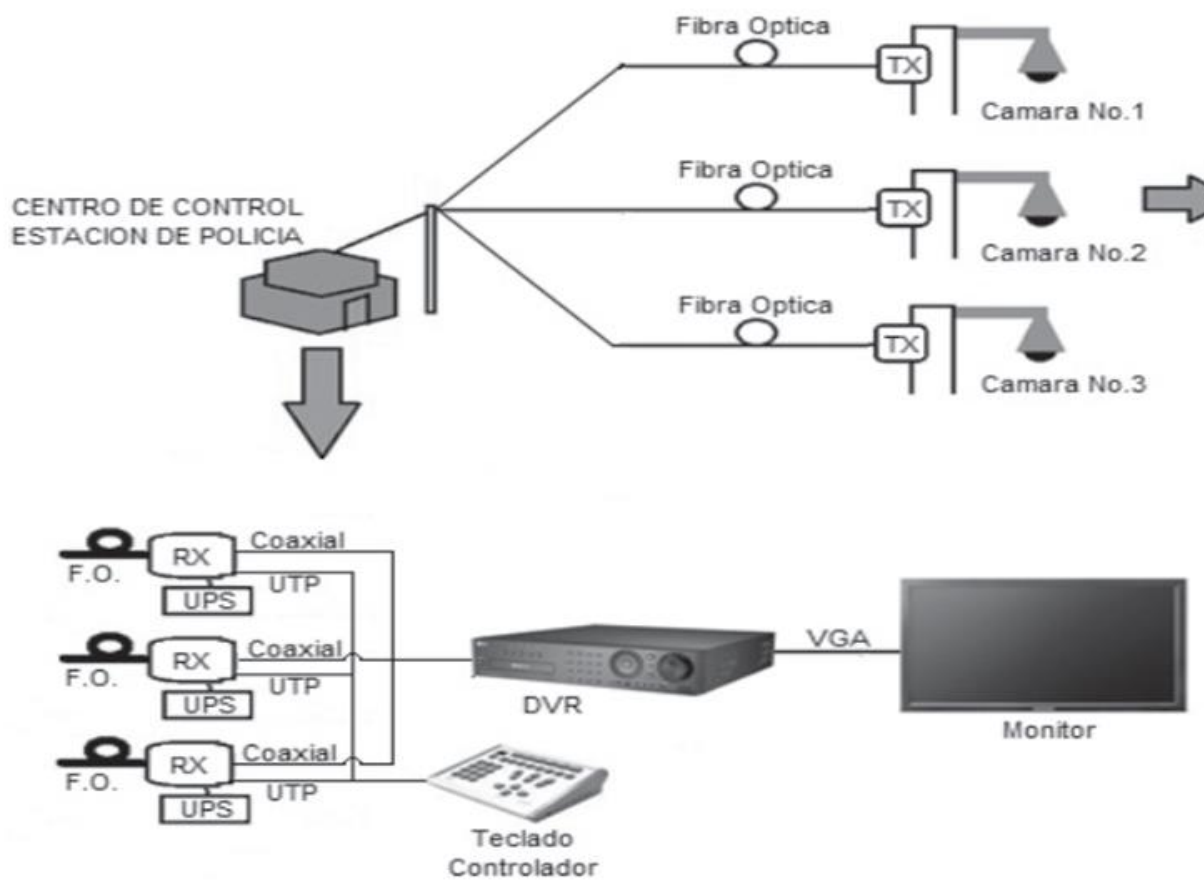

EQUIPO DE POSTERIA

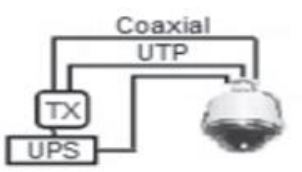

Figura 1. Modelo del sistema resultante

\section{Características Técnicas del Sistema}

Topología de la Red:

Medio de Comunicación:

Tipo de Fibra Óptica:

Protocolo de Comunicación:

Velocidad de Transmisión:

Formato del Video:

Software:

Punto a Punto

Fibra Óptica

Monomodo de 24 Hilos

RS-422

4800 Baudios

MPEG-4

Linux Embebido

Como resultado final se puede observar una topología de red punto a punto, el cual permite tener un sistema confiable en cuanto a daños, debido a que si se presenta un daño en la red para alguna cámara solo esta quedara aislada y el resto del sistema quedara funcionando. Su medio de comunicación es la fibra óptica monomodal de 24 hilos con un ancho de banda de $10 \mathrm{Gbps}$ y un alcance de $20 \mathrm{~km}$ aproximadamente. Todo este sistema se ha sincronizado para el correcto funcionamiento bajo el protocolo de comunicación RS-422 a una velocidad de transmisión de 4800 baudios (por defecto). El formato de video del DVR configurado para la compresión del video es el MPEG-4. El control de cámaras se realiza secuencialmente mediante el teclado controlador. 


\subsection{Pruebas de calidad de la red de fibra óptica}

Se deben realizar pruebas para verificar el estado final de la red de fibra óptica instalada. Estas pruebas fueron realizadas por medio del dispositivo OTDR (Optical Time-Domain Reflectometer). Este dispositivo funciona de tal forma que se le conecta una fibra y este le envía un pulso lumínico el cual va hasta donde sea el final y regresa, permitiendo con esta prueba conocer la distancia de la fibra, el número de empalmes (conexiones) y las pérdidas que existen en el medio.

Esta prueba se realizó desde los tres puntos, los cuales dan los siguientes resultados:

\section{PRUEBA 1: Del centro de control a la cámara $\mathbf{N}^{\circ} 1$}
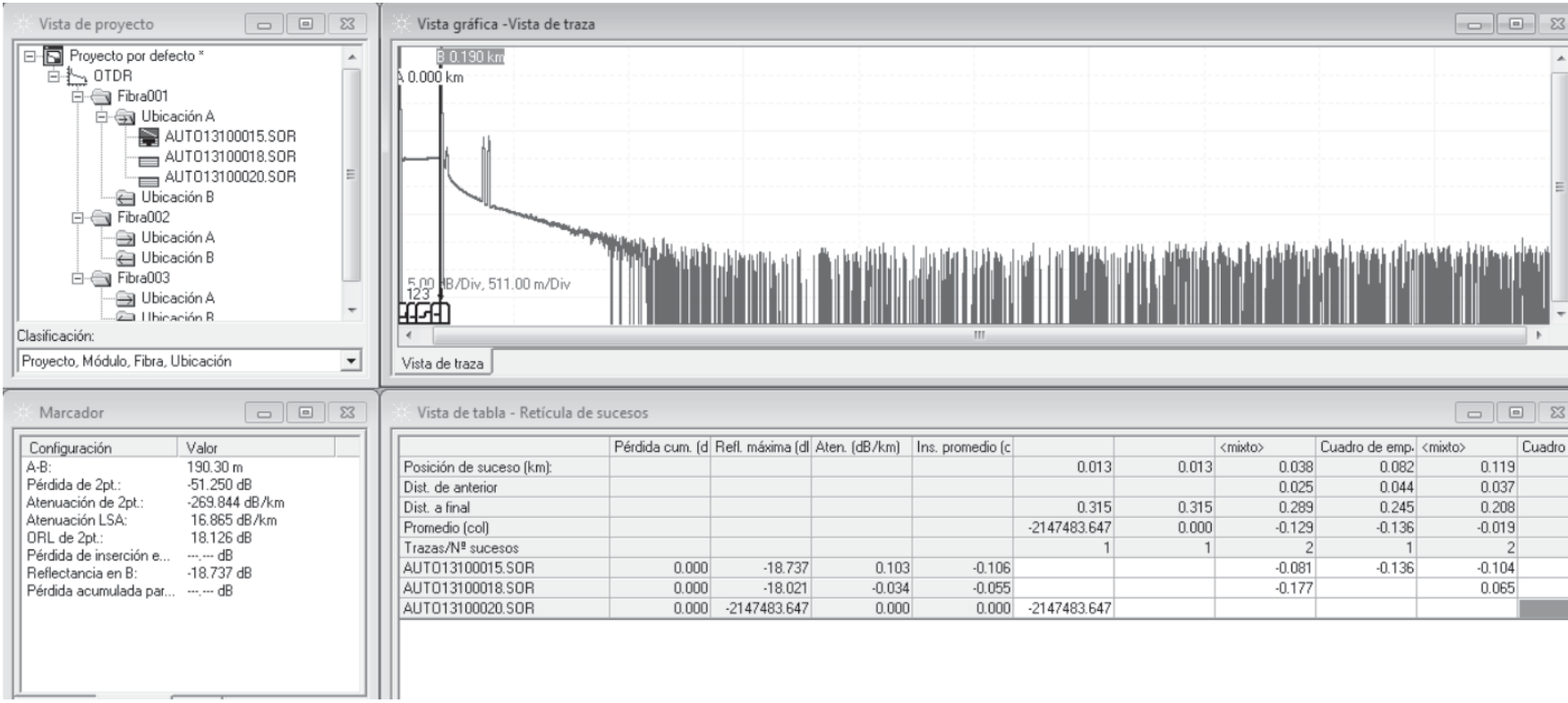

Figura 2. Prueba 1 con el OTDR

\section{PRUEBA 2: Del centro de control a la cámara $\mathbf{N}^{\circ} \mathbf{2}$}

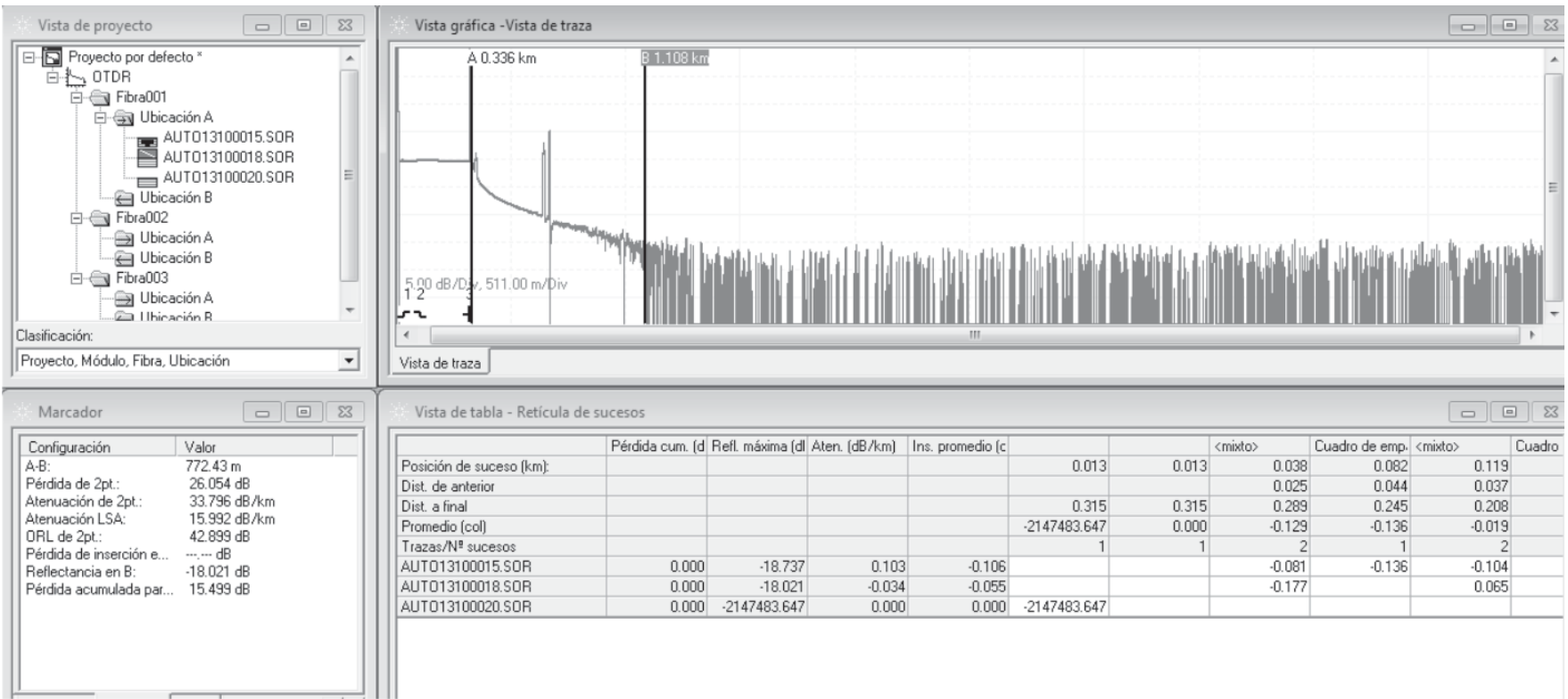

Figura 3. Prueba 2 con el OTDR 


\section{PRUEBA 3: Del centro de control a la cámara $\mathbf{N}^{\circ} \mathbf{3}$}

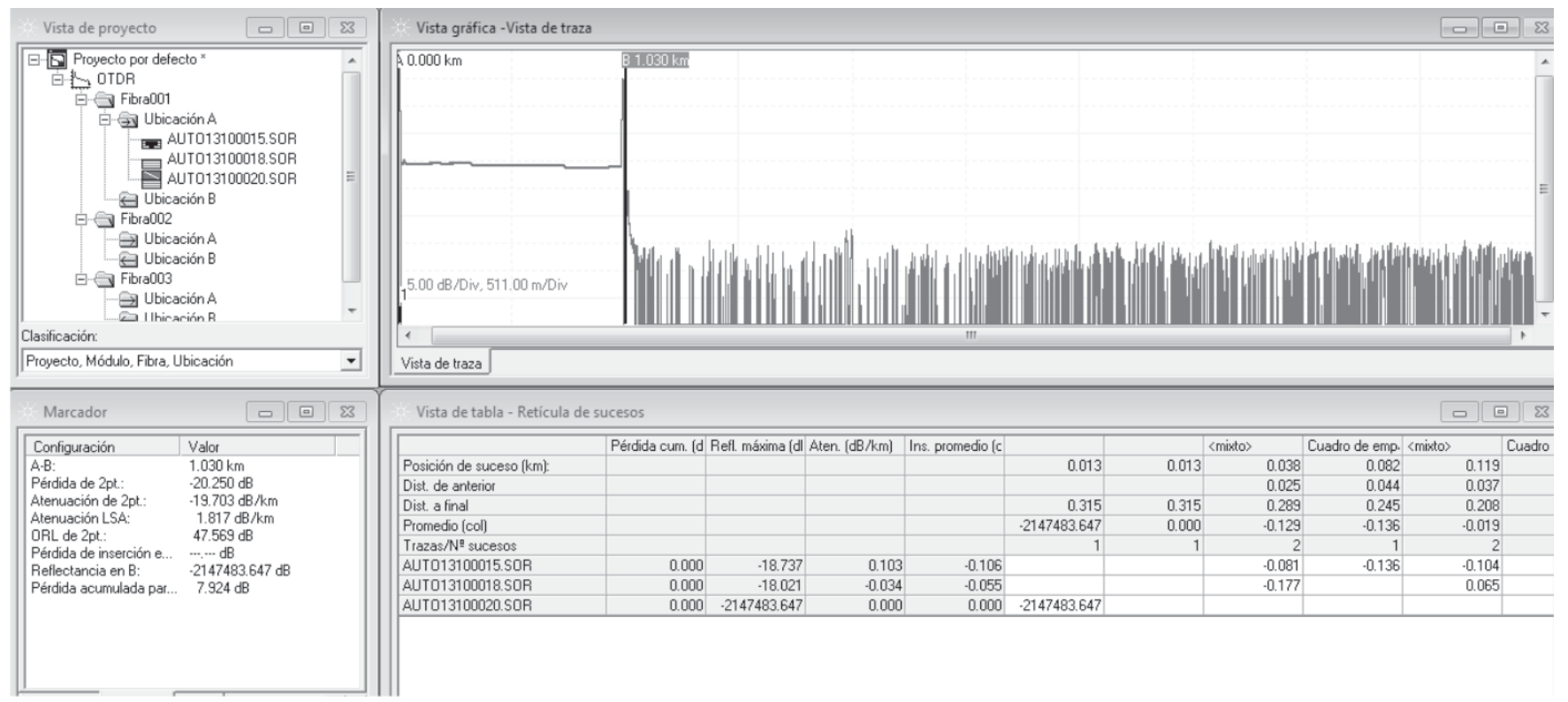

Figura 4. Prueba 3 con el OTDR

Como se puede observar para las tres opciones de mediciones encontramos que parámetros de pérdidas para el balanceo del sistema se logró satisfactoriamente, debido a que las pérdidas por conexión están por debajo de los $0.2 \mathrm{~dB}$, y que las pérdidas totales del sistema están por debajo de los $28 \mathrm{~dB}$ que soportan los transmisores/receptores, ya que esta es su potencia nominal de transmisión.

\subsection{Limitaciones}

Debido que para poder desarrollar un sistema de video vigilancia requiere de mucho dinero, la única limitante que se contó para este sistema fue por recurso financiero. Para dar solución a tal problema la implementación del sistema se divide en dos fases, las cuales se enuncian a continuación:

1). Diseño total del sistema para las 15 cámaras, con la implementación de tres primeras cámaras piloto.

2). La implementación de las restantes 12 cámaras, con la adquisición de sus respectivos equipos de transmisión/ recepción y su debida implementación.

Este sistema cuenta con otra limitante y es que su capacidad total para instalación es de 24 cámaras debido a que el sistema de fibra óptica es de solo 24 hilos.

\section{Conclusiones}

Se sincronizó el sistema de video vigilancia mediante la creación de alarmas en el programa de control del DVR LG para informar la presencia de personas en las horas de la noche, para los lugares donde nadie debe permanecer, y de igual forma se programaron las cámaras para que realizaran recorridos automáticos en las horas del día.

Con la implementación de una red propia de fibra óptica para municipio, permite ampliar el sistema de video vigilancia cuando se desee, o cuando sea el caso del crecimiento del Municipio, debido a que esta red cuenta con gran disponibilidad de ancho de banda.

Se logra que el tráfico de datos sea de forma segura, rápida y de calidad, permitiendo gran hermetismo en las 
comunicaciones para la Policía.

Como aporte al programa Huila Digital se pone a disposición la reserva de la red de fibra óptica, la cual se podrá utilizar a futuro en aplicaciones públicas o privadas como son la prestación del servicio de internet, telefonía IP y televisión digital.

Se logra un resultado final para la Policía de permitir una integración, coordinación y trabajo sinérgico entre la fuerza pública y los organismos de seguridad.

\section{Referencias Bibliográficas}

1. Rojas, J., 2007. Revista sobre la Sociedad del Conocimiento, 5. Mecanismos de Video Vigilancia en la Sociedad de la Información. España, 2-6.

2. Agpd, 2006. Agencia Española de Protección de Datos: Guía de Video Vigilancia. España, 4-36.

3. Barbero J. M., 1999. Comunicación y solidaridad en tiempos de globalización, Encuentro Continental de Comunicadores. Oclacc, Celam, Sertal. Medellín, 20-30.

4. Edward L. S., 1998. Introducción a la Fibra Óptica y el Láser, Ed. Paraninfo, 10-40.

5. Conceptos básicos de la utilización de la fibra óptica, 2000. Por José Isabel Parra Alvarado Secretario General de la Sociedad Astronómica de Aragón Ilhuícat. [Consultado el 8 de febrero de 2010]. http://informatica.aragon. unam.mx/ilhuicatl/fibra.html. 